\title{
Shallow water modeling of Antarctic Bottom Water Crossing the Equator
}

\author{
Paul F. Choboter \\ College of Oceanic and Atmospheric Sciences, Oregon State University, Corvallis, Oregon, USA \\ Gordon E. Swaters \\ Department of Mathematical and Statistical Sciences, University of Alberta, Edmonton, Alberta, \\ Canada
}

[1] The dynamics of abyssal equator-crossing flows are examined by studying simplified models of the flow in the equatorial region in the context of reduced-gravity shallow water theory. A simple "frictional geostrophic" model for one-layer cross-equatorial flow is described, in which geostrophy is replaced at the equator by frictional flow down the pressure gradient. This model is compared via numerical simulations to the one-layer reduced-gravity shallow water model for flow over realistic equatorial Atlantic Ocean bottom topography. It is argued that nonlinear advection is important at key locations where it permits the current to flow against a pressure gradient, a mechanism absent in the frictional geostrophic model and one of the reasons this model predicts less cross-equatorial flow than the shallow water model under similar conditions. Simulations of the shallow water model with an annually varying mass source reproduce the correct amplitude of observed time variability of cross-equatorial flow. The time evolution of volume transport across specific locations suggests that mass is stored in an equatorial basin, which can reduce the amplitude of time dependence of fluid actually proceeding into the Northern Hemisphere as compared to the amount entering the equatorial basin. Observed time series of temperature data at the equator are shown to be consistent with this hypothesis.

\section{Introduction}

[2] In the Atlantic Ocean, the deepest part of the thermohaline circulation consists of Antarctic Bottom Water (AABW), which originates in the Weddell Sea near Antarctica. In this location, particularly cold and fresh water sinks to the bottom of the ocean and flows northward along the sloping ocean floor in the western south Atlantic. While part of this flow remains in the Southern Hemisphere and ultimately mixes upward into shallower waters [Ledwell et al., 2000], part of the flow has been observed to cross the equator into the Northern Hemisphere [DeMadron and Weatherly, 1994; Friedrichs and Hall, 1993]. This current, therefore, acts as a conduit for heat, salt and nutrients to be transported global-scale distances and between hemispheres.

[3] Despite its importance, details of AABW flow remain poorly understood. The qualitative path of AABW is disputed in the literature, since more than one path is consistent with existing 
observations [DeMadron and Weatherly, 1994; Speer and Zenk, 1993; Sandoval and Weatherly, 2001]. While temporal variability of AABW on various timescales has been clearly observed [Hall et al., 1997; Mercier and Speer, 1998; Rhein et al., 1995], the source and nature of this variability is not completely understood.

[4] It is known that AABW enters and exits the Brazil Basin (see Figure 1) through specific channels. The volume flux entering the basin has been measured at $6.9 \times 10^{6} \mathrm{~m}^{3} \mathrm{~s}^{-1}=6.9 \mathrm{~Sv}$ [Hogg et al., 1999]. The amount exiting through the equatorial channel northwest of the basin is 2.0-2.2 Sv [Hall et al., 1997], and the amount exiting through fracture zones in the Mid-Atlantic Ridge to the northeast of the basin is $1.22 \pm 0.25 \mathrm{~Sv}$ [Mercier and Speer, 1998]. The remaining 3.6 Sv of fluid is believed to mix vertically into shallower waters, especially over the rough bathymetry of the Mid-Atlantic Ridge [Ledwell et al., 2000]. The behavior of the current is complicated by the fact that it is believed to split up into two parts with different paths [Sandoval and Weatherly, 2001] and that it is highly variable in time [Hall et al., 1997; Rhein et al., 1995; Mercier and Speer, 1998].

[5] Theoretical and modeling studies of equator-crossing currents are challenging not only because of the breakdown of geostrophy at the equator, but also because potential vorticity is not conserved in these flows [Edwards and Pedlosky, 1998a, 1998b; Nof and Borisov, 1998]. Nof and Borisov [1998] studied the shallow water dynamics of equator-crossing currents in a meridional channel and concluded that the equator-crossing process is an inertial one in which the geometry of the bottom topography plays a crucial role. (Similar conclusions were made by Rodwell and Hoskins [2001] about the atmospheric equator-crossing flow associated with the summer monsoon.) The potential vorticity is modified by friction as the current proceeds, allowing the fluid to flow along the path prescribed by the bottom topography

[6] Johnson [1993] derived an inertial model of abyssal flow valid on an equatorial b plane and over a sloping bathymetry. The analytical solution of this model was used to study the characteristics of bottom water flow crossing the equator in the form of a steady jet with constant potential vorticity flowing along a linear slope. It was found that some upslope motion and fluid acceleration allowed the jet to conserve its transport and potential vorticity as the equator was approached. North of the equator, the jet narrowed, developed a downslope velocity component, and continued to accelerate.

[7] The movement of abyssal waters in the equatorial ocean has also been studied by Stephens and Marshall [2000], who performed numerical simulations of a one-layer model over realistic oceanic bathymetry. This model may be thought of as a simplification of shallow water dynamics where the fluid mass is conserved (a relatively small amount of fluid is lost as it mixes vertically into shallower layers), but momentum balance is reduced to planetary geostrophy with 
Rayleigh drag-type friction. This model will be referred to as a "frictional geostrophic' model. The steady state flow was found to be broadly consistent with observations [Stephens and Marshall, 2000].

[8] The frictional geostrophic (FG) model and the shallow water model were compared for flow over idealized bottom topography by Choboter and Swaters [2000]. Despite the simplicity of the FG model, it captured many of the qualitative aspects of fully nonlinear shallow water dynamics, including the splitting of the flow into northward and southward flowing currents along the eastern slope of a meridional channel. The differences between the shallow water model and the FG model predictions, such as FG model currents along the eastern slope located too close to the bottom of the channel, were attributed to the lack of inertia in the FG model.

[9] Recent research into these abyssal cross-equatorial flows has thus focussed either on studying the nonlinear and time-dependent dynamics of the flow over idealized topography or on examining the steady state flow over realistic equatorial topography as predicted by a simplified model. The FG model has been demonstrated in the literature to be a model that is capable of correctly predicting the qualitative path of abyssal waters. In this paper, we will investigate how well the reduced-gravity shallow water model performs such a task. Since the FG model has already been used in this setting, we will carefully document what the similarities and differences are between the two models.

[10] The role of baroclinicity is investigated by Choboter and Swaters [2003], who derive a twolayer model of abyssal flow. Their model reduces to the subinertial model of Swaters [1991] in the midlatitude limit, yet predicts well defined flow in the equatorial limit. The model exhibits weakened coupling between the two layers in the equatorial limit, which lends support to the neglect of baroclinic effects in the equatorial simulations described here.

[11] The models are intended to simulate AABW flow northward in the southern Atlantic Ocean, so a mass source of dense fluid is introduced at the southern edge of our numerical domain, and it is observed as it flows northward. Two kinds of experiments are performed: steady inflow conditions with an analysis of the steady state conditions, and time-varying inflow conditions to simulate the annual signal seen in observational studies.

[12] The FG model results are compared to the shallow water results and to observations. It is found that, while the FG model predicts qualitatively correct fluid paths, it consistently underpredicts the amount of cross-equatorial mass flux as compared to the shallow water model run with a similar set of parameters. It is shown that the lack of fluid inertia in the FG model prevents the fluid from flowing against a dynamic pressure gradient, significantly restricting the motion. This is consistent with Choboter and Swaters [2000], who found that the FG model did not allow currents to flow as high on idealized topography as the shallow water model. 
[13] Comparison of the shallow water model results to observations shows qualitative agreement with the observed velocity field. The momentum balance at steady state suggests that the nonlinear advection terms not present in the FG model are important as the equatorial basin is entered by the fluid. Time-dependent simulations are described that develop a cross-equatorial mass flux similar in magnitude and time dependence to the observed mass flux. Finally, the in situ temperature data of Hall et al. [1997] are used to support the idea that fluid is temporarily stored in the equatorial basin, which implies that AABWescaping the equatorial basin and proceeding into the Northern Hemisphere the equatorial basin, which implies that AABWescaping the equatorial basin and proceeding into the Northern Hemisphere may do so with a smaller amplitude of annual signal than the AABW flowing into the equatorial channel.

[14] The FG model is described in section 2. The numerical methods used in this study are summarized in section 3. Section 4 presents the results of numerical experiments with steady sources, and section 5 discusses the results of the time-dependent source experiments. The conclusions of this research and related future research are discussed in section 6.

[15] Models in which the momentum equations have been reduced to the geostrophic relations with the addition of a linear term representing the effects of friction have been used to study various large-scale motions by several authors (Stephens and Marshall [2000], Edwards et al. [1998], Samelson [1998], Samelson and Vallis [1997], Salmon [1986], Rhines [1989], and Speer et al. [1993], among others). The FG model may be written

$$
\begin{gathered}
-f v=-g^{\prime} \frac{\partial\left(h+h_{B}\right)}{\partial x}-r u, \\
f u=-g^{\prime} \frac{\partial\left(h+h_{B}\right)}{\partial y}-r, \\
\frac{\partial h}{\partial t}+\nabla \cdot(h \mathbf{u})=0,
\end{gathered}
$$

where $\mathrm{u}=(\mathrm{u}, \mathrm{v})$ is the horizontal velocity, $\mathrm{h}$ is the height of the fluid layer, $h \mathrm{~B}$ is the bottom topography elevation, $\mathrm{g} 0$ is the reduced gravity, $\mathrm{f}$ is the variable Coriolis parameter, and $\mathrm{r}$ is a damping coefficient to be specified.

[16] Speer et al. [1993] employ a similar model to investigate the effects of topography and strong layer thickness variation on bottom layer flow. They focus on Northern Hemisphere flow and include in their model the effect of entrainment of fluid into the motionless upper layer, 
which leads to thinning of the active layer and, in some cases, grounding of the layer interface. Entrainment is neglected here since we focus not on basin-scale dynamics, but rather upon the motion of $\mathrm{AABW}$ within a few degrees of latitude of the equator. The neglect of entrainment in the northwestern Brazil Basin is consistent with evidence that upward mixing of abyssal waters in the Brazil Basin depends strongly on geographical position, with much of it achieved near the rough topography of the Mid-Atlantic Ridge [Ledwell et al., 2000].

[17] The FG model as stated above does not parameterize the effects of friction in a realistic way. However, it does have some justification in terms of vorticity. The potential vorticity equation of this model is

$$
\frac{\partial}{\partial t}\left(\frac{f}{h}\right)+\mathbf{u} \cdot \boldsymbol{\nabla}\left(\frac{f}{h}\right)=-\frac{r}{h} \zeta,
$$

where $\varsigma=\delta v / \delta x-\delta u / \delta y$ is the relative vorticity. Therefore this model neglects relative vorticity in favor of planetary vorticity, and it simulates the dissipation of potential vorticity by Ekman friction. In that sense, the effect of a bottom Ekman layer is similar to the effect of linear Rayleigh friction with a coefficient $r=\left(\mathrm{V}_{\mathrm{z}} f / 2\right)^{1 / 2} / \mathrm{h}=f \partial_{\mathrm{E}} / 2 h$, where $v_{z}$ is a vertical eddy viscosity coefficient, $\partial_{\mathrm{E}}$ is the Ekman layer thickness, and $\mathrm{h}$ is the layer thickness [Pedlosky, 1987;

Stephens and Marshall, 2000; Kawase and Straub, 1991]. This implies that $r \rightarrow 0$ at the equator. In order to allow friction to be nonzero at the equator, Stephens and Marshall [2000] neglect the dependence of $\mathrm{r}$ on $\mathrm{f}$, but retain the $\mathrm{h}$ dependence, as do Speer et al. [1993]. However, Edwards et al. [1998], Samelson [1998], and Samelson and Vallis [1997] all neglect the h dependence as well, taking $r$ to be a prescribed constant. In the present study, $\mathrm{r}$ is assumed independent of $f$ and $h$.

[18] Modeling friction effects with Rayleigh damping terms has the advantage of allowing the velocities to be solved for in a diagnostic relation in terms of the pressure gradients:

$$
u=g^{\prime} \frac{-f p_{y}-r p_{x}}{f^{2}+r^{2}}, \quad v=g^{\prime} \frac{f p_{x}-r p_{y}}{f^{2}+r^{2}}
$$

where $p=h+h_{B}$ and subscripts with respect to $x$ and $y$ denote the respective partial derivatives. Clearly, the singularity at $f=0$ present in the geostrophic relations has been removed as long as $r$ $\neq 0$. One consequence of equation (5) is that the component of velocity parallel to the pressure gradient is necessarily in the direction opposite to that gradient. From equation (5), 


$$
\begin{aligned}
\mathbf{u} \cdot \boldsymbol{\nabla} p & =g^{\prime} \frac{-f p_{y}-r p_{x}}{f^{2}+r^{2}} p_{x}+g^{\prime} \frac{f p_{x}-r p_{y}}{f^{2}+r^{2}} p_{y} \\
& =-\frac{g^{\prime} r}{f^{2}+r^{2}}\left(p_{x}^{2}+p_{y}^{2}\right) \leq 0 .
\end{aligned}
$$

Geometrically, $\mathrm{u} \cdot \boldsymbol{\nabla} \mathrm{p} \leq 0$ means the velocity and pressuregradient vectors have an angle $\mathrm{q} p / 2$ between them. Fluid may flow down a pressure gradient, but never up a pressure gradient.

[19] Although $r \neq 0, r$ is considered to be a small parameter. The potential vorticity equation (4) may be investigated to see the behavior this model predicts in the small $r$ parameter regime. In the limit as $r \rightarrow 0$, equation (4) states that $f / h$ is conserved following the flow. This implies that, for small $r$ (to be precise, $\mathrm{r}$ is assumed to be small relative to the largest value of $\mathrm{f}$, i.e., $0<r \ll$ $\max |f|$ ), a mass of fluid approaching the equator will tend to decrease in height, since $|f|$ decreases as the equator is approached. This process continues until the right-hand side of equation (4) becomes nonnegligible, which is when $r / h \sim O(1)$ (nondimensional values). This represents the point at which the effects of friction will be dynamically important. Under the assumption that $\mathrm{h}$ and $\mathrm{f}$ are each $O(1)$ initially, frictional effects will thus be important at a latitude where $f=\mathrm{O}(r)$; that is, at a nondimensional distance from the equator of $r / \beta$, where $\beta=$ $\delta \mathrm{f} / \delta \mathrm{y}$ at $y=0$. With $r=0.05, \beta=1$ and $L=200 \mathrm{~km}$, this corresponds to a dimensional distance of $10 \mathrm{~km}$.

[20] The evolution of potential energy in this model may be expressed in the following form:

$$
\frac{\partial}{\partial t}\left(\frac{1}{2} g h^{2}+g h h_{B}\right)+\nabla \cdot(\Phi h \mathbf{u})=-r h \mathbf{u} \cdot \mathbf{u} .
$$

Potential energy, $(1 / 2) g h^{2}+g h h_{\mathrm{B}}$, is not, of course, conserved in this model, but decreases with time. It appears that the rate of potential energy dissipation is simply proportional to the damping parameter r; however,

$$
\begin{aligned}
\mathbf{u} \cdot \mathbf{u} & =\frac{\left(g^{\prime}\right)^{2}\left[\left(-f p_{y}-r p_{x}\right)^{2}+\left(f p_{x}-r p_{y}\right)^{2}\right]}{\left(f^{2}+r^{2}\right)^{2}} \\
& =\frac{\left(g^{\prime}\right)^{2}}{f^{2}+r^{2}}\left[p_{x}^{2}+p_{y}^{2}\right]
\end{aligned}
$$


so that the potential energy dissipation is only proportional to $\mathrm{r}$ as long as $f \gg r$. Very near the equator, where $f \ll r$ the velocity is proportional to $1 / r$, and thus the dissipation of potential energy is actually proportional to $1 / r$ there.

3. Numerical Methods

[21] The shallow water model may be written in nondimensional form as

$$
\begin{gathered}
\frac{\partial \mathbf{u}}{\partial t}+\mathbf{u} \cdot \boldsymbol{\nabla u}+\frac{f}{R o} \mathbf{k} \times \mathbf{u}=-\frac{1}{R o} \boldsymbol{\nabla}\left(h+h_{B}\right)+\mathbf{F}_{\text {fric }} \\
\frac{\partial h}{\partial t}+\boldsymbol{\nabla} \cdot(\mathbf{u} h)=0,
\end{gathered}
$$

where $\mathbf{u}$ is the horizontal velocity vector, $F_{\text {fric }}$ represents the friction term (see equation (10) below for the specific form of friction used), $R o=U / f_{0} L$ is the Rossby number, and $U$, $L, f_{0}$, and $h_{0}$ are typical scales for the velocity, length, Coriolis parameter and fluid depth, respectively. It has been assumed that the time variable is scaled advectively, $T=L / U$, for a timescale $T$, and that the scale slope for the bottom topography is the same as the scale slope of the fluid height, $h_{0} / L$. We have also assumed the geostrophic scaling $f_{0} U=g^{\prime} h_{0} / L$, i.e., $U^{2} /\left(g^{\prime} h 0\right)=R o$.

[22] The velocity scale $U$ has been taken to be $5 \mathrm{~cm} \mathrm{~s}^{-1}$. (This is consistent with observational data; see DeMadron and Weatherly [1994], Rhein et al. [1995], Sandoval and Weatherly [2001], and Hall et al. [1997].) The Coriolis parameter has been scaled by its value at $5^{\circ} \mathrm{N}$, and the horizontal length scale $L$ is $200 \mathrm{~km}$. This gives a Rossby number (at $5^{\circ} \mathrm{N}$ or $5^{\circ} \mathrm{S}$ ) of $R o=0.0197$, and a timescale of $T=46.3$ days, i.e., 1 year is 7.88 nondimensional time units. The Coriolis parameter varies realistically with latitude. Nondimensionally,

$$
f=\sin [\theta(y)] / \sin 5^{\circ}
$$

where the latitude, $\theta$, is expressed as a function of y by $\theta=L y / R$, and $R=6371 \mathrm{~km}$ is the Earth's equatorial radius. The magnitude of $R o$ implies that the flow will be geostrophically balanced to leading order at a latitude of $5^{\circ} \mathrm{N}$ or $5^{\circ} \mathrm{S}$. The scale depth of the fluid layer is $h_{0}=400 \mathrm{~m}$. Reduced gravity is set to $g^{\prime}=3.2 \times 10^{-4} \mathrm{~m} \mathrm{~s}^{-1}$.

[23] These equations are discretized on an Arakawa C grid [Arakawa and Hsu, 1990]. The spatial discretization of the advection, Coriolis and pressure gradient terms is performed using the scheme of Arakawa and Hsu [1990]. This scheme, which is designed to tolerate an arbitrarily small layer thickness (i.e., intersections of the fluid interface with the bottom topography), 
conserves energy and weakly dissipates potential enstrophy when the mass flux is nondivergent (neglecting friction and any errors introduced by the time-stepping routine). It is a second-order accurate scheme in space.

[24] The temporal discretization of the momentum equations is done according to a third-order accurate scheme due to Matsuno [1966]. It is a third-order Runge-Kutta method.

[25] The mass equation is stepped forward in time using the method of Hsu and Arakawa [1990], which is a predictor-corrector scheme second-order accurate in time and space that maintains the positive definiteness of the height field $\mathrm{h}$ and conserves mass.

[26] To integrate the frictional geostrophic model numerically, the same routine as for the full shallow water equations is used for the mass equation, but since there is no time derivative to evaluate in the velocity relations of equation (5), these are simply evaluated at each time step using second-order accurate centered differences for the derivatives.

[27] Dirichlet boundary conditions are used for h, u, and v. Except at locations along the boundary where fluid is flowing into the domain, no-slip and no-flux conditions are imposed by setting $\mathrm{h}=0, \mathrm{u}=0$, and $\mathrm{v}=0$ on the boundary. Where fluid enters the domain, $\mathrm{h}$ is specified as discussed below, the velocity component parallel to the domain boundary is zero, and the velocity component normal to the domain boundary is set to the geostrophic velocity.

[28] Where fluid is flowing into the domain, the height at the boundary is set to have a cosine profile:

$$
h= \begin{cases}\frac{h_{\max }}{2}\left[1+\cos \left(\frac{\pi R}{R_{\max }}\right)\right] & \text { for } 0 \leq R \leq R_{\max } \\ 0 & \text { elsewhere }\end{cases}
$$

where $R=\left|x-x_{c}\right|$ and $\mathrm{x}_{\mathfrak{c}}, h_{\max }$ and $R_{\max }$ are specified parameters corresponding to the center, height and the half-width of the inflow profile, respectively. This height profile was chosen so that the height field is continuous and differentiable, to assist with numerical stability. Unless otherwise specified, the inflow has a height of 0.7 (280 meters) and a half-width of $0.7(140 \mathrm{~km})$. The resulting rate of mass flow into the domain depends upon the local topographic slope, since that will affect the geostrophic velocity. In each of the runs reported here, the mass inflow rate is $5 \mathrm{~Sv}$. 
[29] The fluid exits the domain at the eastern and northern boundaries. To minimize the effects of the numerical boundaries on the interior of the fluid, the velocity and height of the fluid are damped within a sponge layer next to these boundaries. The damping factor decreases smoothly from unity next to the interior of the fluid to zero at the boundary. The velocity and height are multiplied by this damping factor after each time step, thus providing a mass sink within the sponge layer before the fluid reaches the numerical boundary. The sponge layer extends 21 grid points in from the boundary, which is a dimensional distance of $200 \mathrm{~km}$. Therefore the physical domain of the simulations extends only to the inner edge of the sponge layer; eastward to $26.05^{\circ} \mathrm{W}$ longitude and northward to $3.58^{\circ} \mathrm{N}$ latitude. (The entire numerical domain is shown in Figure 2.)

[30] We have taken friction to be of the form

$$
\mathbf{F}_{\text {fric }}=A_{H} \nabla^{2} \mathbf{u}+A_{N} \nabla^{6} \mathbf{u}-A_{V} \frac{\mathbf{u}}{h^{2}},
$$

where $A_{H}, A_{N}$, and $A_{V}$ are the horizontal, numerical, and vertical friction coefficients, respectively. For the simulations reported here $A_{H}=5 \times 10^{-3}, A_{N}=10^{-6}$, and $A_{V}=10^{-5}$, which correspond to dimensional values of $A^{*}{ }_{H}=50 \mathrm{~m}^{2} \mathrm{~s}^{-1}, A^{*}{ }_{N}=1.6 \times 10^{19} \mathrm{~m}^{6} \mathrm{~s}^{-1}$, and $A^{*} \mathrm{v}=4 \times 10^{-7}$ $\mathrm{m}^{2} \mathrm{~s}^{-1}$, respectively, on a grid with $9.5 \mathrm{~km}$ resolution. The horizontal friction term is intended to represent the effects of horizontal diffusion of momentum by subgrid-scale eddies. The numerical and vertical friction terms are added for numerical stability. Numerical friction effectively removes small-scale features, and vertical friction is added only to prevent the unphysical acceleration of massless grid points. The horizontal and numerical terms are evaluated at each of the three time levels in the Matsuno [1966] scheme, but the vertical term is fully implicit at each time step.

[31] It should be emphasized that we are not attempting to accurately model the dynamics of friction. In fact, given the simple form of friction and the parameters used, the shallow water simulations will certainly be more frictional than abyssal flows in nature, and friction will turn out to be nonnegligible in certain parts of the domain. However, it will be shown that friction does not dominate the dynamics over most of the path of the abyssal current. It will also be argued that the differences between the FG model and shallow water model are not that one is frictional and the other is inertial, but rather, that the absence of inertial terms in the FG model leads to singular behavior of the fluid. The lack of any flow whatsoever up the pressure gradient in the FG model limits the dynamics significantly.

[32] Note that the numerical scheme of Arakawa and Hsu [1990] tolerates arbitrarily small thicknesses, but not a thickness of exactly zero, since potential vorticity $q=(z+f) / h$ must be 
explicitly calculated. Thus we maintained a minimum nonzero height field at all grid points. This was set to a nondimensional value of $10^{-5}$ (dimensionally, $4 \mathrm{~mm}$ ). Smaller values led to increased numerical instability, and larger values led to poorer conservation of kinetic and potential energy.

[33] The bathymetry used in the numerical simulations is based upon the Naval Oceanographic Office's digital bathymetric database. The bathymetric data are projected onto a $148 \times 253$ grid and are smoothed by six iterations of replacing a data point with the nine-point average of the data surrounding it. This averaging was judged by eye to be a reasonable compromise between removing grid-scale features and retaining large-scale features of the topography. The dimensional resolution of the grid is $9.5 \mathrm{~km}$, so this smoothing removed features approximately on the order of $40 \mathrm{~km}$ or less.

\section{Steady Source Results}

[34] The shallow water model and the FG model were run to steady state. Steady state was determined to have been reached when the output diagnostic values of potential and kinetic energy approached steady values. (Typically, at steady state the diagnostic variables were varying by less than $1 \%$ of their value over one nondimensional time unit.) The shallow water model retained some time variability after reaching quasi-steady-state, so quantities have been time-averaged over 6 months after reaching quasi-steadystate.

[35] For both models, the flow is observed to follow bathymetric contours quite closely (Figure 3 ). While the entire current initially flows northward along the contours, part of the flow turns northwest and enters the equatorial basin, which is located between $1^{\circ} \mathrm{S}$ and $1^{\circ} \mathrm{N}$ and between $34^{\circ} \mathrm{W}$ and $38^{\circ} \mathrm{W}$ and part of the flow turns east and exits the numerical domain. However, the FG model predicts less flow into the equatorial basin, and ultimately, across the equator.

[36] Qualitatively, both models seem to predict the southern- intensified flow within the equatorial basin that was observed by Hall et al. [1997]. One notable difference between the steady flow fields is the jet of fluid at $2.5^{\circ} \mathrm{S}$ latitude, $32.5^{\circ} \mathrm{W}$ longitude present in the shallow water simulation but not the FG simulation. Another difference is in the activity of fluid in the equatorial basin. The shallow water model predicts meanders there while the FG model exhibits motion only along the southern edge of the basin.

[37] The mass transport of this current is measured for each of these simulations across five locations (see Figure 2 and Table 1). The measured fluxes are (a) the flow into the domain, (b) the flow eastward out of the domain, (c) the flux into the equatorial basin from the Brazil Basin, (d) the flux across the mooring locations of Hall et al. [1997] (hereinafter referred to as HMW), and (e) the flux out of the equatorial basin into the Northern Hemisphere. 
[38] The transports measured at these locations at steady state for each of the models is also listed in Table 1. Each model predicts that the majority of fluid does not enter the equatorial basin, but exits the domain eastward (3.6 Sv exits eastward in FG, 3.4 exits eastward in shallow water). The shallow water model predicts $1.4 \mathrm{~Sv}$ across the HMW mooring line, but the frictional geostrophic model predicts a flow of only $0.96 \mathrm{~Sv}$ there. These measured fluxes along with the transport stream function lines displayed in Figure 3 are suggestive that, since uphill flow is not possible in the FG model, the only fluid successfully crossing the equator is fluid that was initially at shallow enough depths to flow over the ridges located before and after the equatorial basin.

\subsection{Parameter Dependences}

[39] A suite of simulations have been performed. For brevity, we report in detail here only on the simulations with realistic values of cross-equatorial AABW transport. However, it must be noted that this transport depends strongly upon two parameters in particular: the position of the Southern Hemisphere mass source and the damping parameter $r$ in the FG model. These parameter dependences are indicated in Figure 4.

[40] The position of mass source along the topography was varied, and the resulting flux across the equator measured at steady state. In Figure 4a, the depth of the inflow current is defined to be the position relative to the ocean surface of the center of mass of the influx profile, as calculated by numerically integrating over the region of inflow for each of the different current locations. For both models, the equator-crossing flux depends on the initial depth of the current, with the FG model consistently predicting less equator-crossing flow than the shallow water model at the same initial current position.

[41] Since the downhill component of flow in the FG model also depends upon the damping coefficient, one would expect that coefficient to affect the amount of AABW crossing the equator. The damping parameter was varied, and the percentage flux crossing the equator measured at steady state. The results are shown in Figure 4b. An increase in the damping parameter leads to a decrease in the amount of fluid crossing the equator. The damping parameter is set to $\mathrm{r}=0.02$ for the simulations reported here. Since the velocity at $\mathrm{f}=0$ is proportional to $1 / \mathrm{r}$ (see equation (7)), very small damping parameters can lead to high velocities at the equator, causing numerical instability.

\subsection{Momentum Balances}

[42] The values of the terms in the shallow water momentum equations at steady state are displayed in Figure 5. The sign convention for momentum terms is taken such that a positive term acts as an accelerating force in the positive $\mathrm{x}$ or $\mathrm{y}$ direction (eastward or northward, respectively); that is, the dimensional momentum equations are expressed in the form 


$$
\frac{\partial \mathbf{u}}{\partial t}=-f \mathbf{k} \times \mathbf{u}-g^{\prime} \nabla\left(h+h_{B}\right)-\mathbf{u} \cdot \nabla \mathbf{u}+\mathbf{F}_{\text {fric }}
$$

and the four terms on the right-hand side are referred to as Coriolis, pressure gradient, advection, and friction, respectively.

[43] The balance of momentum is geostrophic over much of the domain, with friction important near the fluid edges (Figure 5). Friction in this run is dominated by the hyperdiffusion term, $A_{N} \nabla^{6} \mathbf{u}$. Where friction is important, it takes on a small-scale banded structure. This small-scale structure is seen also in the pressure gradient, implying that the balance near the edge of the fluid is between the pressure gradient and friction. The fact that these small-scale structures do not appear in the Coriolis term is evidence that the velocity field does not vary strongly on these small scales.

[44] The nonlinear advection term becomes important at isolated locations, most notably as the fluid is about to enter the equatorial basin. Specifically, at $33^{\circ} \mathrm{W}, 2^{\circ} \mathrm{S}$, the zonal component of advection is negative (westward), while the pressure gradient is eastward there. The Coriolis term is westward. The velocity at that location has a westward component (since the $y$ component of Coriolis is negative, see also Figure 3). Thus this is an example of fluid inertia enabling the fluid to flow against the pressure gradient, which cannot happen in the FG model.

[45] The momentum terms are looked at again in Figure 6, where 93 points are chosen along one of the streamlines that cross the equator. At each of these points, the two components of each momentum term are projected onto the local along-streamline and across-streamline directions. In the along-streamline direction, the main balance is between the pressure gradient and advection. Note in particular the positive advection driving the flow against a negative pressure gradient between points 10 and 25 . The across streamline plot shows that the flow is in geostrophic balance before point 25. After that point, the pressure and Coriolis terms are weaker, and the remaining terms make a greater contribution to the balance. As the flow crosses the equator near point 80 , all terms appear relatively weak, followed by a strong increase in the pressure gradient and friction in both components as the fluid flows over the ridge at the northwest corner of the equatorial basin.

[46] The momentum terms may also be calculated for the FG model, although there are no advection terms and the friction is simply Rayleigh drag. Despite the simplicity of the FG model, the momentum terms exhibit significant spatial variability (Figure 7). Friction does not appear to be limited to the boundaries of the fluid, but the dominant balance is geostrophic over much of the domain, especially south of $2^{\circ} \mathrm{S}$. 
[47] The FG momentum terms were broken down into along- and across-streamline components along the 96 points shown in Figure 8. The FG model is not discretized using its momentum equations (1) and (2), but instead with the form where $u$ and $v$ have been isolated, equation (5). On the Arakawa $\mathrm{C}$ grid, the terms in equation (5) with coefficient $\mathrm{f}$ in the numerator must be calculated on a larger stencil than the other terms. As a result of this, the momentum terms calculated individually do not necessarily balance exactly, with the largest errors in the Coriolis terms. This can be seen in Figure 8 where the along-stream Coriolis term is not zero. While the along-stream Coriolis term suffers from understood errors, the remaining terms are meaningful as plotted.

[48] Note that the terms are an order of magnitude smaller than their shallow water counterparts. The along-stream balance is between a weak pressure gradient and friction. Note that the pressure gradient is positive or near zero along the whole streamline. The cross-stream balance is geostrophic, since friction is exactly zero in the cross-stream direction.

\section{Time-Dependent Source}

[49] HMW measured a mean equator crossing flux of about 2.0 Sv, but also observed a significant time variability in the AABW flow in the equatorial basin (Figure 9). They measured changes in the observed transport on the order of $4 \mathrm{~Sv}$ in a few days, varying from as much as 5 Sv northward to $2 \mathrm{~Sv}$ southward over the 604 day data set. The 40 day averaged transport was slightly greater than $2 \mathrm{~Sv}$ northward for much of the year, but was close to zero at the same time of year (beginning of March) 2 years in a row. This implies that the AABW has an annual signal associated with it, with magnitude on the order of the total volume flux itself.

[50] To evaluate the ability of the shallow water model and the FG model to capture this time variability, simulations were performed with time-varying inflow conditions, and the flux was measured as a function of time across the five positions noted in Figure 2. The model was first run out to steady state, then the strength of the Southern Hemisphere mass source was varied sinusoidally in time with a period of 1 year.

[51] The simulated volume flux measured at the HMW mooring locations have an annual maximum at between 2 and $4 \mathrm{~Sv}$ and an annual minimum effectively at zero (Figure 10). This is roughly consistent with the observations of HMW (Figure 9). However, the volume flux of AABW into the domain does not drop below $1 \mathrm{~Sv}$ at any time. The FG model run under the same conditions predicts similar behavior: the volume flux across the HMW moorings varies from a maximum of $2 \mathrm{~Sv}$ to a minimum of $0 \mathrm{~Sv}$, while the AABW source never is less than $1 \mathrm{~Sv}$. Assuming these simulations to be relevant to the flow of $\mathrm{AABW}$ in the real ocean, this would imply that the flow of AABW through the Brazil Basin need not drop to zero for the flow of $\mathrm{AABW}$ through the equatorial basin to be zero. 
[52] Note also that the simulated flow of AABW into the Northern Hemisphere from the equatorial basin also does not drop to zero in either the shallow water or the FG simulations, even though the flow through the middle of the equatorial basin does momentarily vanish. This may be explained by the idea that the fluid is temporarily stored in the equatorial basin for part of the annual cycle. Figures 10 and 11 also show the flow at the southern entrance to the equatorial basin developing a significant negative flux for part of the year. During the time of lowest AABW flux into the domain, fluid is spilling out of both ends of the equatorial basin!

[53] The transports in both Figures 10 and 11 show a time delay from when the source of fluid enters the domain to when the effect of the change in source is detected at the various flux lines. The information entering the domain propagates as a wave along the topography. An order of magnitude estimate of its phase speed in these simulations is $10 \mathrm{~km} /$ day.

[54] The hypothesis that mass is stored in the equatorial channel may be tested by comparing the time-dependent simulated AABW thickness with the thickness of the AABW implied by the temperature data of HMW (Figure 12). Following HMW, we assume the top of the AABW is at the $1.8^{\circ} \mathrm{C}$ isotherm, and we assume a linear temperature gradient. HMW measured the temperature gradient near $4100 \mathrm{~m}$ depth to be $-4.03 \times 10^{-3}{ }^{\circ} \mathrm{C} \mathrm{m}^{-1}$ at the beginning of their data collection and $-3.56 \times 10^{-30} \mathrm{C} \mathrm{m}^{-1}$ at the end. We use the mean of these values. Given that the depth of the ocean at this location is $4536 \mathrm{~m}$, we convert from the temperature $\mathrm{q}$ at a depth of $4093 \mathrm{~m}$ to thickness of the AABW $\mathrm{h}$ via

$$
h=443+\frac{1.8-\theta}{3.795 \times 10^{-3}} .
$$

thickness is in reasonable agreement with the observed values. The shallow water model does a particularly good job, while the FG model seems to underpredict the AABW thickness by $\sim 10 \%$. One feature the simulations do not capture well is the maintaining of a relatively large thickness until just before the time of minimum transport. The simulations instead predict a time dependence that is closer to sinusoidal.

[56] The simulated height in the numerical experiments is in phase with the observed height (Figure 13), increasing in height when the mass flux is a maximum, and decreasing in height when the mass flux is at a minimum. This is consistent with the idea that temporary mass storage takes place in the equatorial basin.

\section{Summary}

[57] The frictional geostrophic (FG) model studied here parameterizes frictional and other ageostrophic effects into a Rayleigh damping term. This model has been used instudies of largescale flow, including abyssal equator-crossing flow. We have investigated the usefulness of this model by comparing its predictions to the predictions of shallow water theory over realistic topography. 
[58] Both the FG model and the shallow water model predicted that the cross-equatorial mass flux depends sensitively on the location of the mass source on the slope. The amount of fluid crossing the equator also depended strongly on the damping parameter $r$ of the FG model. Both models were able to reproduce the observed southern-intensified flow within the equatorial basin, although the FG model predicted a much thinner jet located along the south of the equatorial basin with a nearly motionless interior, which is not entirely consistent with observations.

[59] The momentum balance analysis showed that the dominant balance outside the equatorial basin is geostrophic balance, except near the edges of the fluid, where the pressure gradient was in balance with friction. At specific locations before the fluid enters the equatorial basin, nonlinear advection was argued to drive the fluid up a local pressure gradient, increasing the flow into the equatorial basin. The FG model has no advection, which is why it consistently predicted less cross-equatorial flow than the shallow water model under similar conditions.

[60] In the simulations with a time-dependent mass source in the Southern Hemisphere, the time dependence of AABW flux through the equatorial basin was shown to be consistent with the observed values, which are greater than $2 \mathrm{~Sv}$ at maximum and appear to vanish at minimum. This time dependence was achieved in the simulations even though the mass source of AABW entering the domain did not at any time have a volume flux less than $1 \mathrm{~Sv}$. Also, the simulated volume flux of AABW entering the Northern Hemisphere from the equatorial basin did not at any time vanish, even when the flow through the center of the equatorial basin was zero or even slightly negative. This implies that mass may be temporarily stored in the equatorial basin for part of the annual cycle.

[61] The height of simulated AABW was compared with the measured AABW, as calculated from the temperature time series of Hall et al. [1997]. It was found that the simulated height was consistent with observations. It was also found that the simulations predicted the correct phase of the AABW thickness relative to the time of minimum cross-equatorial flux, and that the simulations and the data were consistent with temporary mass storage of AABW in the equatorial basin. This implies that, in the real ocean, the amplitude of time variation of AABW flux out of the equatorial basin may not be as great as the measured time variation of AABW within the equatorial basin.

[62] Adding more reality to the simulations described here would likely involve parameterizations of other AABW mass sinks in the Brazil Basin, including upwelling and flow through the fracture zone in the northeast corner of the Brazil Basin. Adding more shallow layers of active fluid would be an important step, although it would be complicated by the presence of the North Atlantic Deep Water flowing immediately above AABW, and in the opposite direction. 
[63] It must also be noted that de Verdie're and Schopp [1994] established that the "horizontal component' of the Coriolis force, which is neglected as part of the hydrostatic approximation, may be important in equatorial dynamics. This will be true particularly if the horizontal length scales of motion are smaller than $\left(H r_{0}\right)^{1 / 2}$, where $\mathrm{H}$ is a vertical scale of the motion and $r_{0}$ is the radius of the earth. For a vertical scale of $H=200 \mathrm{~m}$, this length scale is on the order of $40 \mathrm{~km}$, which is smaller than the $O(200 \mathrm{~km})$ length scales we study here, and thus the traditional approximation was retained.

[64] The need for comparison between the two models was initially motivated by the question of whether the equatorial abyssal balance is essentially frictional or inertial. The main result of the comparison is that the shallow water model simulated the equator-crossing process better than the FG model. It is true that the FG model correctly reproduced several observed features. The flow was southern intensified in the equatorial basin. The correct order of magnitude of crossequatorial transport was predicted. The FG model even reproduced some time-dependent effects seen in the shallow water model, such as the temporary mass storage effects in the equatorial basin. However, the FG model predicts flow paths that are too southern intensified within the equatorial channel. In fact, the moorings of HMW were located along the $36^{\circ} \mathrm{W}$ Longitude line between the $4000 \mathrm{~m}$ bathymetric contours, so they would have measured zero AABW flux if the AABW flowed along the path prescribed by the FG model! The shallow water model also did better than the FG model at predicting the time-dependent height of the fluid in the equatorial channel, as compared with observations.

[65] However, we cannot therefore conclude that the cross-equatorial flow is completely inertial. The shallow water simulations were not purely inertial. The friction terms were not negligible over certain parts of the domain, including parts along the equator-crossing streamline. The important distinction between the two models is that the lack of inertial terms in the FG model severely restricts the motion of the fluid, whereas the shallow water model has inertial terms available when needed. This is entirely consistent with the conclusions of Nof and Borisov [1998] (and Rodwell and Hoskins [2001], for atmospheric equatorcrossing flows), who found that equator-crossing flow is primarily an inertial process, with the topography interacting strongly with the flow, but the presence of friction was necessary to modify potential vorticity as the fluid moved between hemispheres.

[66] Acknowledgments. We thank Mindy Hall for providing us with the postscript files containing Figures 6 and 8 of HMW as well as their temperature time series data. P.F.C. gratefully acknowledges the funding received from the Natural Sciences and Engineering Research Council (NSERC) of Canada in the form of their PGS B scholarship, and from the Killam Foundation in the form of the Killam Memorial Ph.D. Scholarship. Partial support was provided by NSF grant OCE990-7854. G.E.S. gratefully acknowledges continued support by 
NSERC in the form of ongoing research grants. Two anonymous reviewers provided helpful suggestions that significantly improved the paper.

\section{References}

Arakawa, A., and Y.-J. G. Hsu (1990), Energy conserving and potentialenstrophy dissipating schemes for the shallow water equations, Mon. Weather Rev., 118, 1960- 1969.

Choboter, P. F., and G. E. Swaters (2000), Modeling equator-crossing currents on the ocean bottom, Can. Appl. Math. Quart., 8, 367-385.

Choboter, P. F., and G. E. Swaters (2003), Two-layer models of abyssal equator-crossing flow, J. Phys. Oceanogr., 33, 1401- 1415.

DeMadron, X. D., and G. Weatherly (1994), Circulation, transport and bottom boundary layers of the deep currents in the Brazil Basin, J. Mar. Res., 52, 583-638.

de Verdie're, A. C., and R. Schopp (1994), Flows in a rotating spherical shell: The equatorial case, J. Fluid Mech., 276, 233-260.

Edwards, C. A., and J. Pedlosky (1998a), Dynamics of nonlinear crossequatorial flow. Part I: Potential vorticity transformation, J. Phys. Oceanogr., 28, 2382- 2406.

Edwards, C. A., and J. Pedlosky (1998b), Dynamics of nonlinear crossequatorial flow. Part II: The tropically enhanced instability of the western boundary current, J. Phys. Oceanogr., 28, 2407- 2417.

Edwards, N. R., A. J. Willmott, and P. D. Killworth (1998), On the role of topography and wind stress on the stability of the thermohaline circulation, J. Phys. Oceanogr., 28, 756- 778.

Friedrichs, M. A. M., and M. M. Hall (1993), Deep circulation in the tropical North Atlantic, J. Mar. Res., 51, 697- 736.

Hall, M. M., M. McCartney, and J. A. Whitehead (1997), Antarctic Bottom Water flux in the equatorial western Atlantic, J. Phys. Oceanogr., 27, 1903- 1926.

Hogg, N. G., G. Siedler, and W. Zenk (1999), Circulation and variability in the southern boundary of the Brazil Basin, J. Phys. Oceanogr., 29, 145-157.

Hsu, Y.-J. G., and A. Arakawa (1990), Numerical modeling of the atmosphere with an isentropic vertical coordinate, Mon. Weather Rev., 118, 1933- 1959.

Johnson, G. C. (1993), A deep inertial jet on a sloping bottom near the equator, Deep Sea Res., 40, 1781-1792.

Kawase, M., and D. Straub (1991), Spinup of source-driven circulation in an abyssal basin in the presence of bottom topography, J. Phys. Oceanogr., 21, 1501- 1514. 
Ledwell, J. R., E. T. Montgomery, K. L. Polzin, L. C. St. Laurent, R. W. Schmitt, and J. M. Toole (2000), Evidence for enhanced mixing over rough topography in the abyssal ocean, Nature, 403, 179- 182.

Matsuno, T. (1966), A finite difference scheme for time integrations of oscillatory equations with second order accuracy and sharp cut-off for high frequencies, J. Meteorol. Soc. Jpn., 44, 85-88.

Mercier, H., and K. G. Speer (1998), Transport of bottom water in the Romanche Fracture Zone and the Chain Fracture Zone, J. Phys. Oceanogr., 28, 779-790.

Nof, D., and S. Borisov (1998), Inter-hemispheric oceanic exchange, Q. J. R. Meteorol. Soc., 124, 2829- 2866.

Pedlosky, J. (1987), Geophysical Fluid Dynamics, 2nd ed., Springer-Verlag, New York.

Rhein, M., L. Stramma, and U. Send (1995), The Atlantic Deep Western Boundary Current: Water masses and transports near the equator, J. Geophys. Res., 100, 2441-2457.

Rhines, P. B. (1989), Deep planetary circulation and topography: Simple models of midocean flows, J. Phys. Oceanogr., 19, 1449- 1470.

Rodwell, M. J., and B. J. Hoskins (2001), Subtropical anticyclones and summer monsoons, J. Clim., 14, 3192-3211.

Salmon, R. (1986), A simplified linear ocean circulation theory, J. Mar. Res., 44, 695- 711.

Samelson, R. M. (1998), Large-scale circulation with locally enhanced vertical mixing, J. Phys. Oceanogr., 28, 712-726.

Samelson, R. M., and G. K. Vallis (1997), A simple friction and diffusion scheme for planetary geostrophic basin models, J. Phys. Oceanogr., 27, 186- 194.

Sandoval, F. J., and G. L. Weatherly (2001), Evolution of the deep western boundary current of Antarctic Bottom Water in the Brazil Basin, J. Phys. Oceanogr., 31, 1440- 1460.

Speer, K., and W. Zenk (1993), The flow of bottom water into the Brazil Basin, J. Phys. Oceanogr., 23, 2667-2682.

Speer, K., E. Tziperman, and Y. Feliks (1993), Topography and grounding in a simple bottom layer model, J. Geophys. Res., 98, 8547-8558.

Stephens, J. C., and D. P. Marshall (2000), Dynamical pathways of Antarctic Bottom Water in the Atlantic, J. Phys. Oceanogr., 30, 622-640. 
Swaters, G. E. (1991), On the baroclinic instability of cold-core coupled density fronts on a sloping continental shelf, J. Fluid Mech., 224, 361-382.

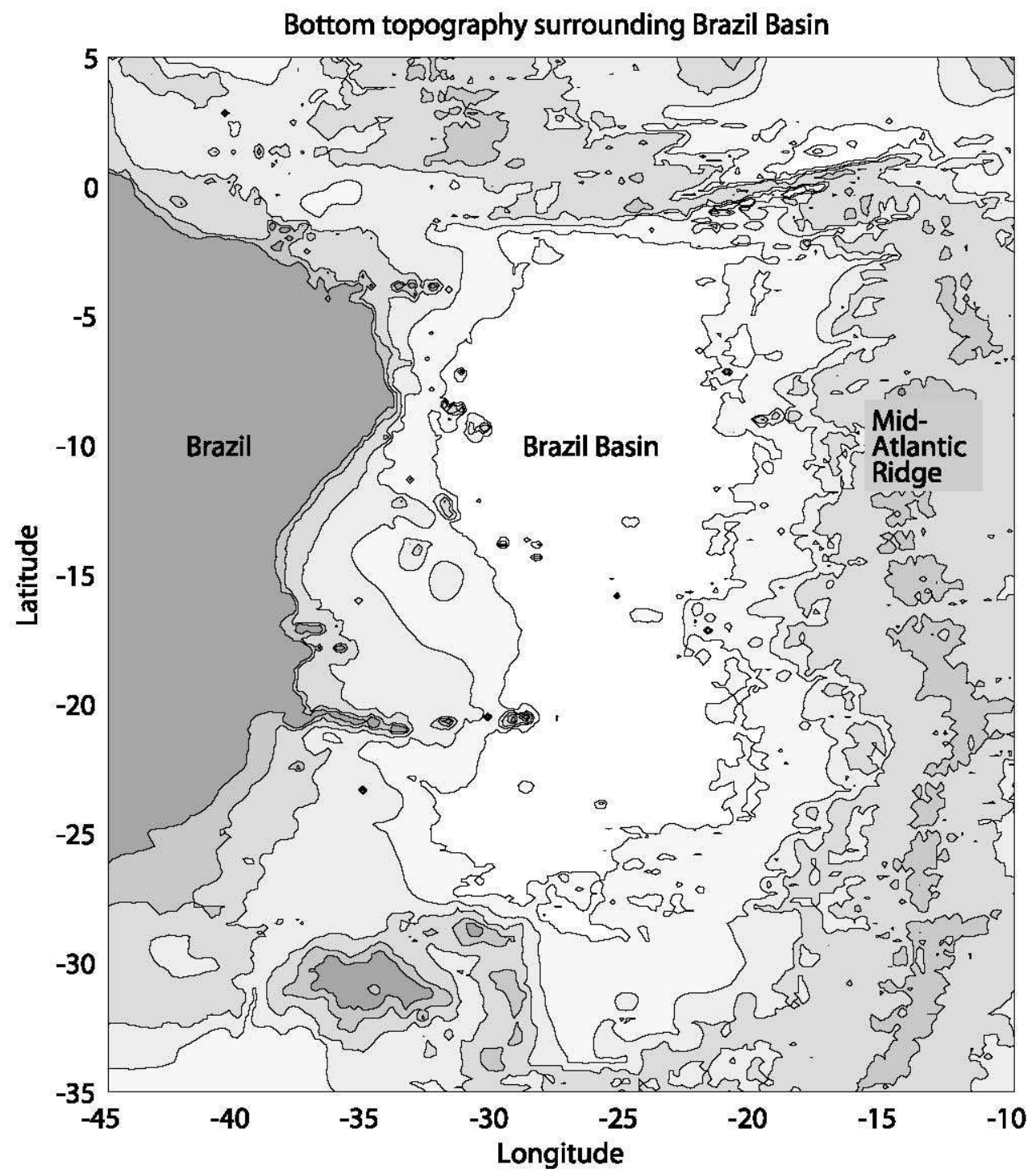

Figure 1. Ocean bottom topography surrounding the Brazil Basin. Contour levels are at depths of 2000 , $3000,4000,4500$, and $5000 \mathrm{~m}$. 


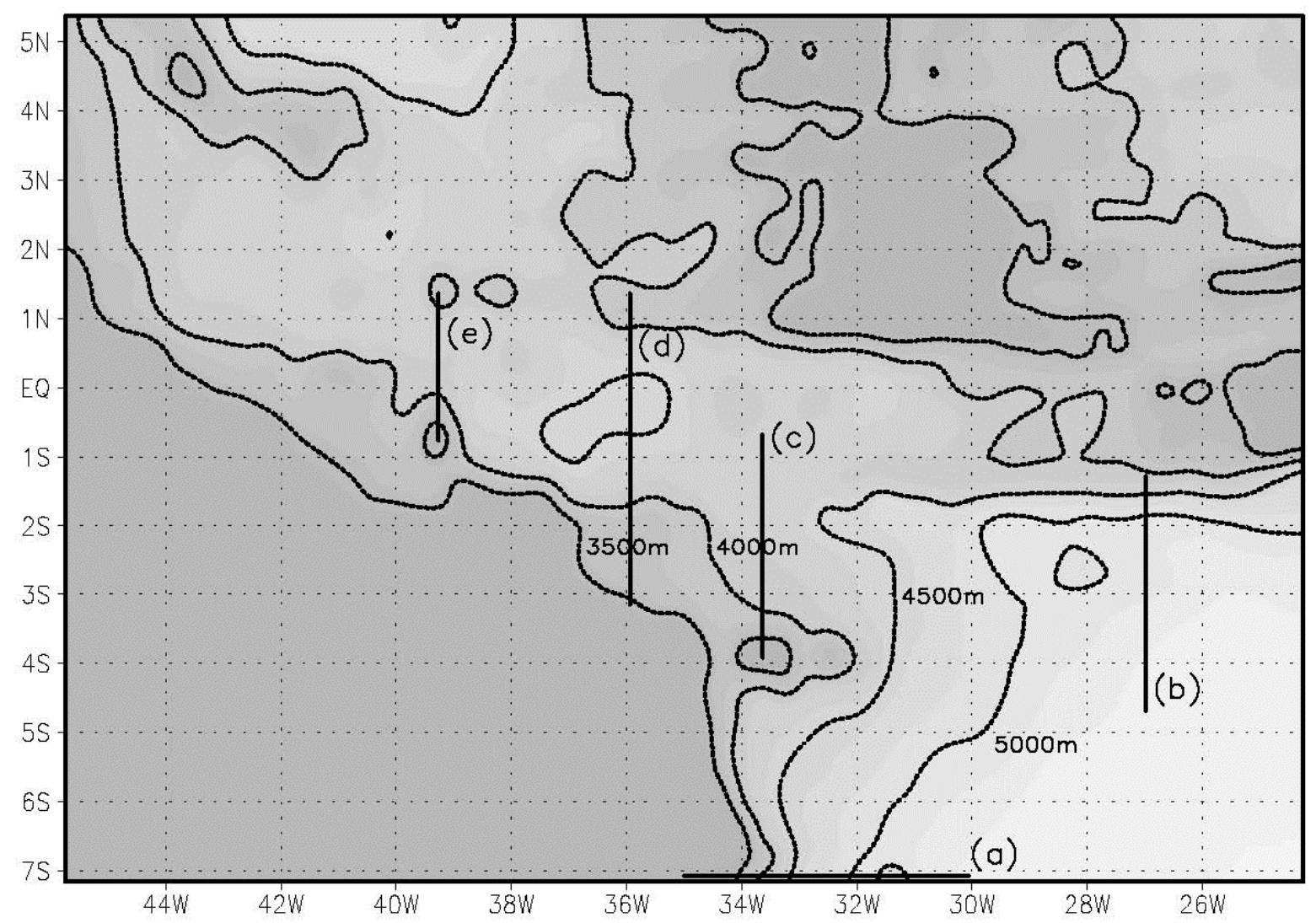

Figure 2. Numerical domain and topography. The shading interval is $200 \mathrm{~m}$. Bathymetric data are from the Naval Oceanographic Office Data Warehouse at https://idbms.navo.navy.mil and have been smoothed prior to use in the simulations. The five lines labeled a-e are the lines across which the flux is measured in our simulations. 


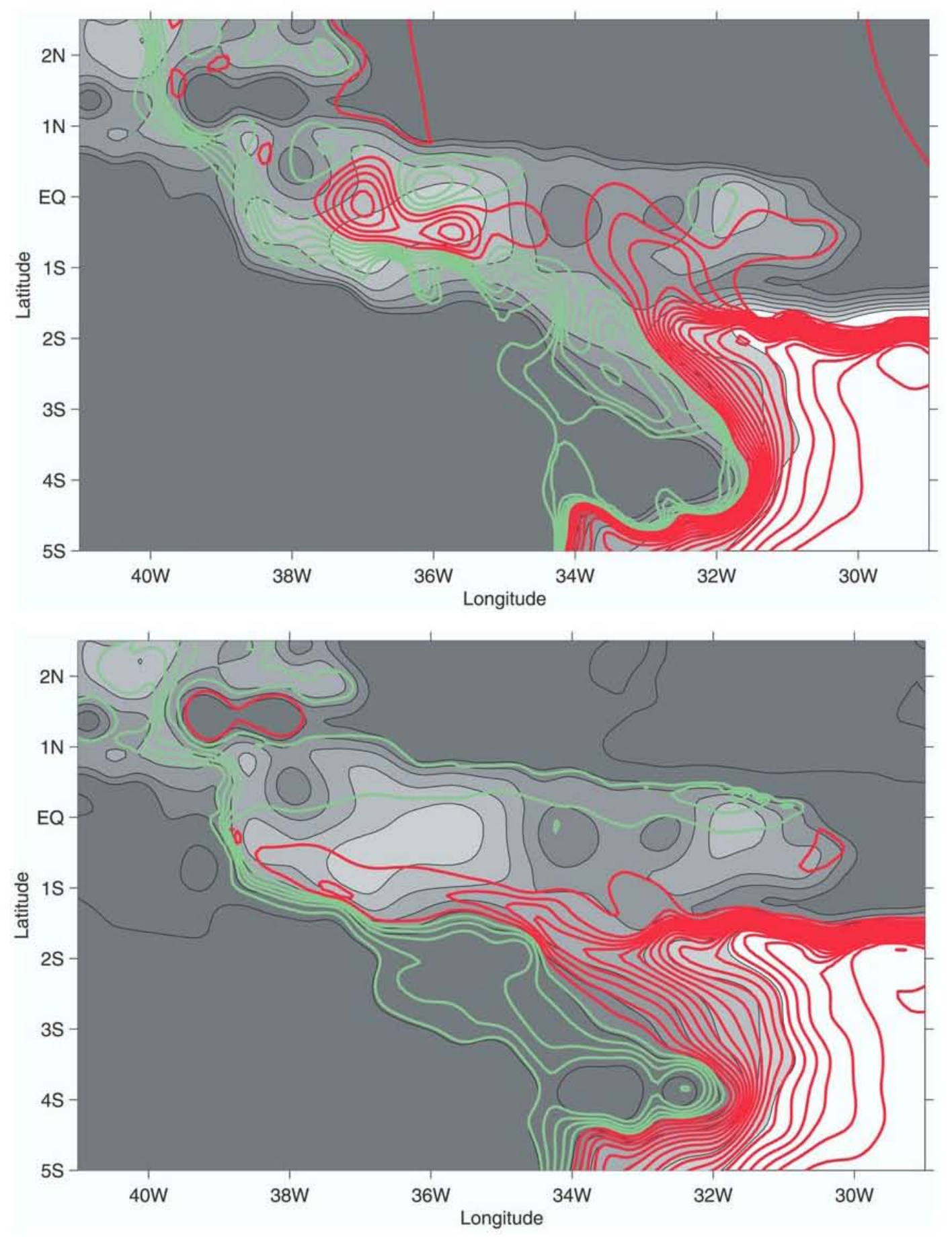

Figure 3. Transport stream function at steady state: (top) shallow water model and (bottom) frictional geostrophic (FG) model. Bathymetry is in gray scale (shallower is darker), with contour lines every $100 \mathrm{~m}$ between $4100 \mathrm{~m}$ and $4600 \mathrm{~m}$. The $3600 \mathrm{~m}$ contour has been added to the FG plot. The shallow water plot is a 6 month mean. Transport contour intervals are $0.2 \mathrm{~Sv}$. Contours of source fluid successfully crossing the equator are green, and remaining contour levels are red. The direction of flow is as follows: green is on the left and red is on the right, facing downstream. The two red contours over the mid-Atlantic ridge in the Figure 3 (top) are the same contour level; there is no net transport between them. 
Table 1. Lines Across Which the Flux is Calculated in the Simulations and Steady State Flux Values for Each Model $^{\mathrm{a}}$

\begin{tabular}{ccccccc}
\hline Line Label & Description & Location, deg & Direction & Range, deg & Shallow Water & FG \\
\hline a & into domain & $7 \mathrm{~S}$ & north & $35-30 \mathrm{~W}$ & 4.99 & 5.19 \\
b & out of domain & $27 \mathrm{~W}$ & east & $4.7-1.3 \mathrm{~S}$ & 3.37 & 3.60 \\
c & into equatorial basin & $33.6 \mathrm{~W}$ & west & $3.9-0.7 \mathrm{~S}$ & 1.14 & 0.83 \\
d & HMW moorings & $35.9 \mathrm{~W}$ & west & $3.1 \mathrm{~S}-1.3 \mathrm{~N}$ & 1.42 & 0.96 \\
e & out of equatorial basin & $39.2 \mathrm{~W}$ & west & $0.8 \mathrm{~S}-1.3 \mathrm{~N}$ & 1.30 & 1.19 \\
\hline
\end{tabular}

${ }^{a}$ The shallow water values are calculated as a 6 month mean. FG, frictional geostrophic model; HMW, Hall et al. [1997].
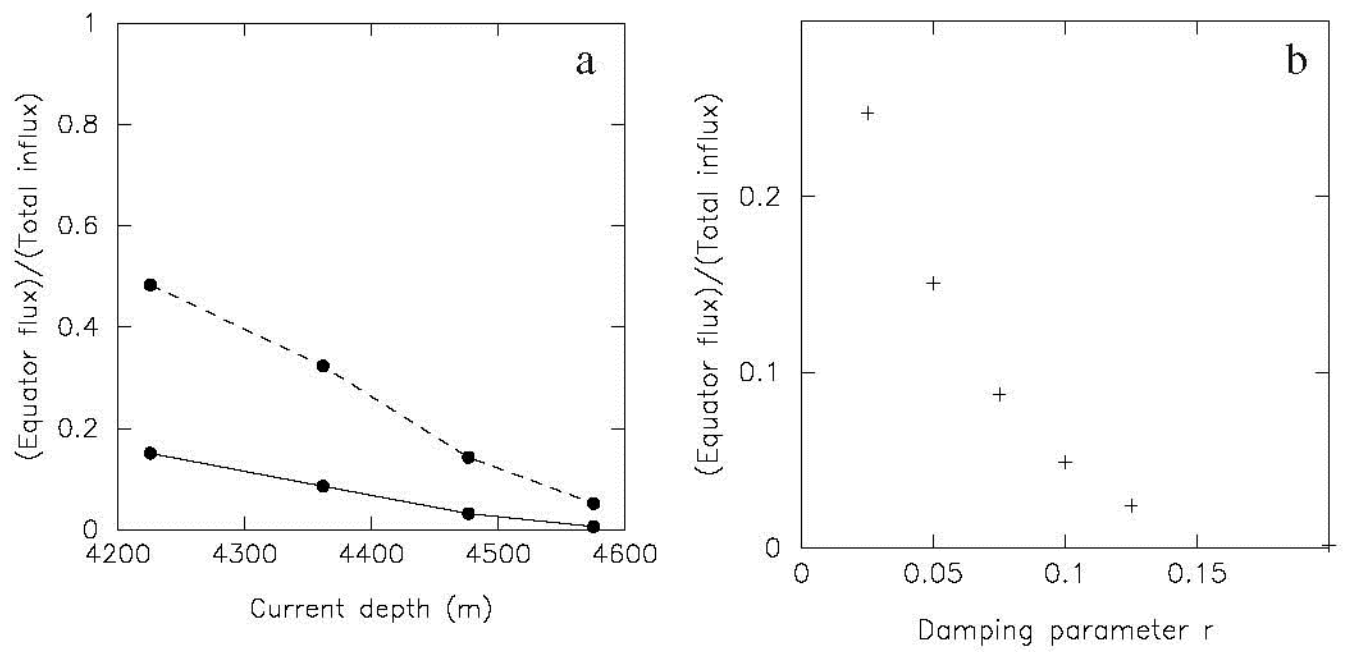

Figure 4. Parameter dependences in the simulations. (a) Flux across the equator as a function of inflow position. The dotted line corresponds to shallow water simulations and the solid line to the FG model. (b) Percentage flux across the equator as a function of the damping parameter in the FG model. In depthvarying runs $r=0.1$ and in $r$-varying runs, inflow depth is $4477 \mathrm{~m}$. In all other simulations, $r=0.02$, and inflow depth is $4363 \mathrm{~m}$. 


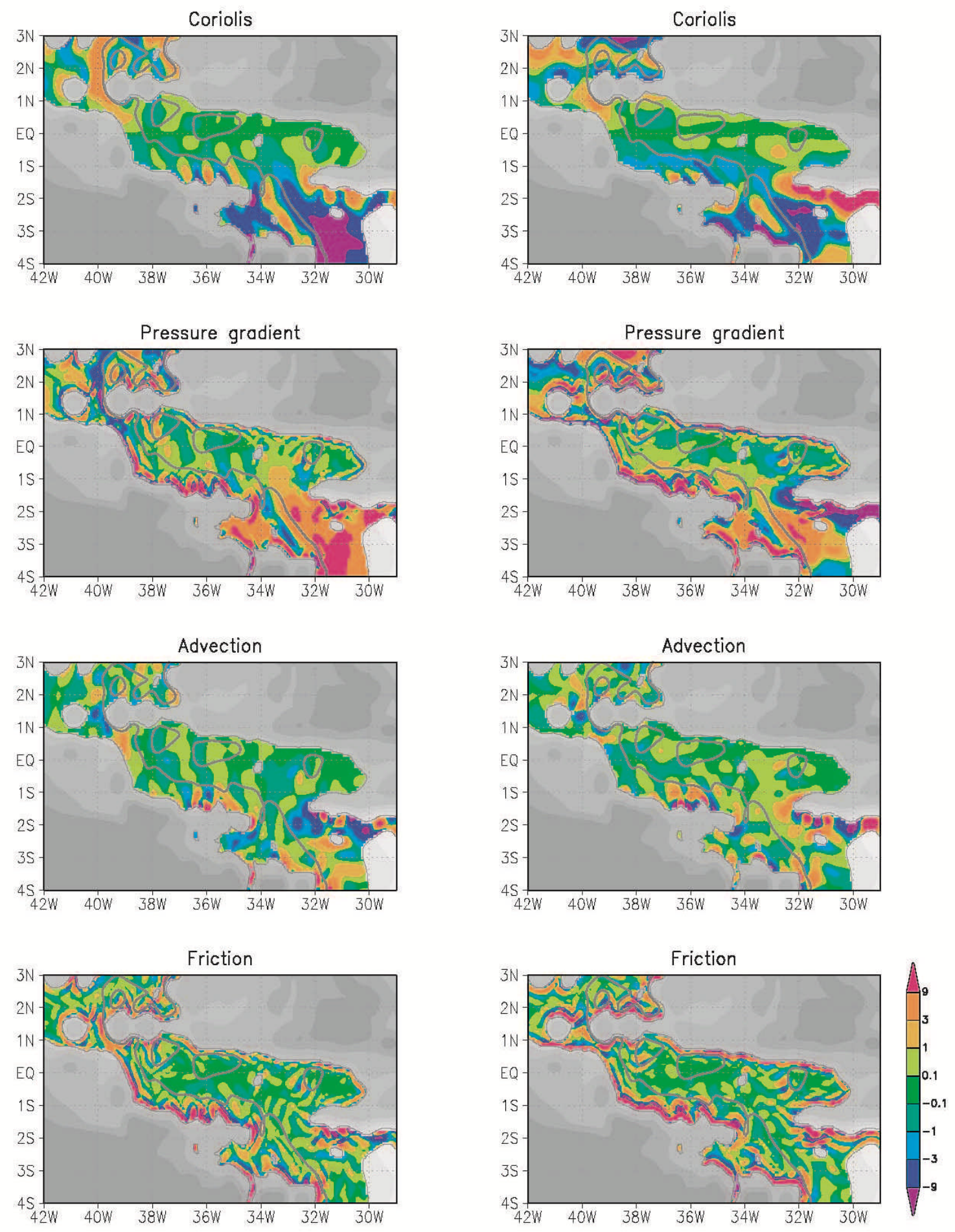

Figure 5. Momentum balances for the shallow water simulation (6 month mean). (left) Zonal component. (right) Meridional component. Positive values represent an accelerating force in the eastward or northward direction. Nonuniform contour spacing is used to reveal features on different scales. Contours are the same for all plots, in units of $10^{-7} \mathrm{~m} \mathrm{~s}^{-2}$. A transport stream function contour line has been added to all plots for cross reference. 

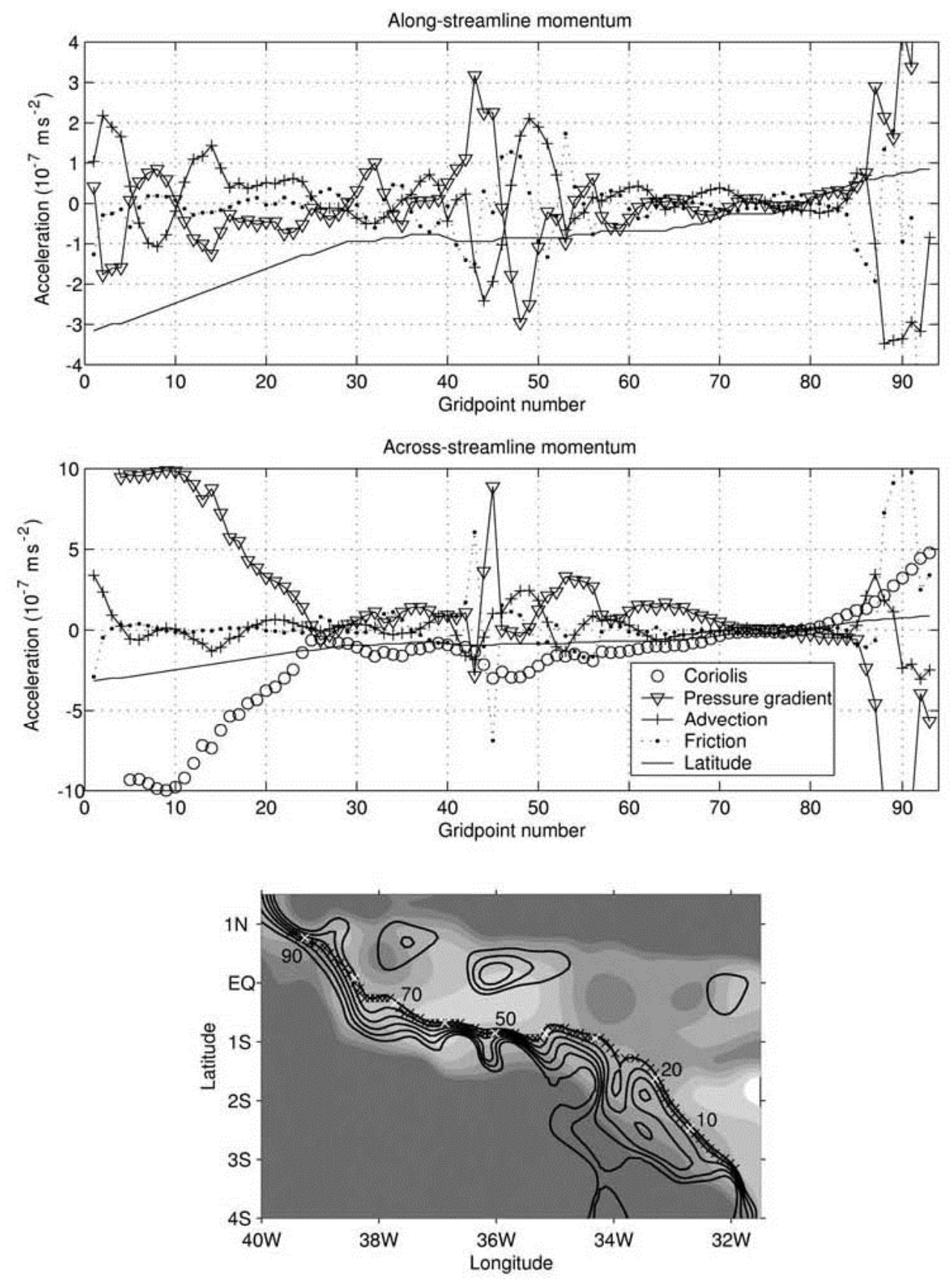

Figure 6. Momentum terms along a streamline, shallow water model. At each point the momentum terms are projected onto the along- and across-streamline directions. A positive quantity in the alongstream direction represents a driving force. A positive quantity in the across-stream plot represents a force pushing to the right. Along-stream Coriolis is zero and is not plotted. 

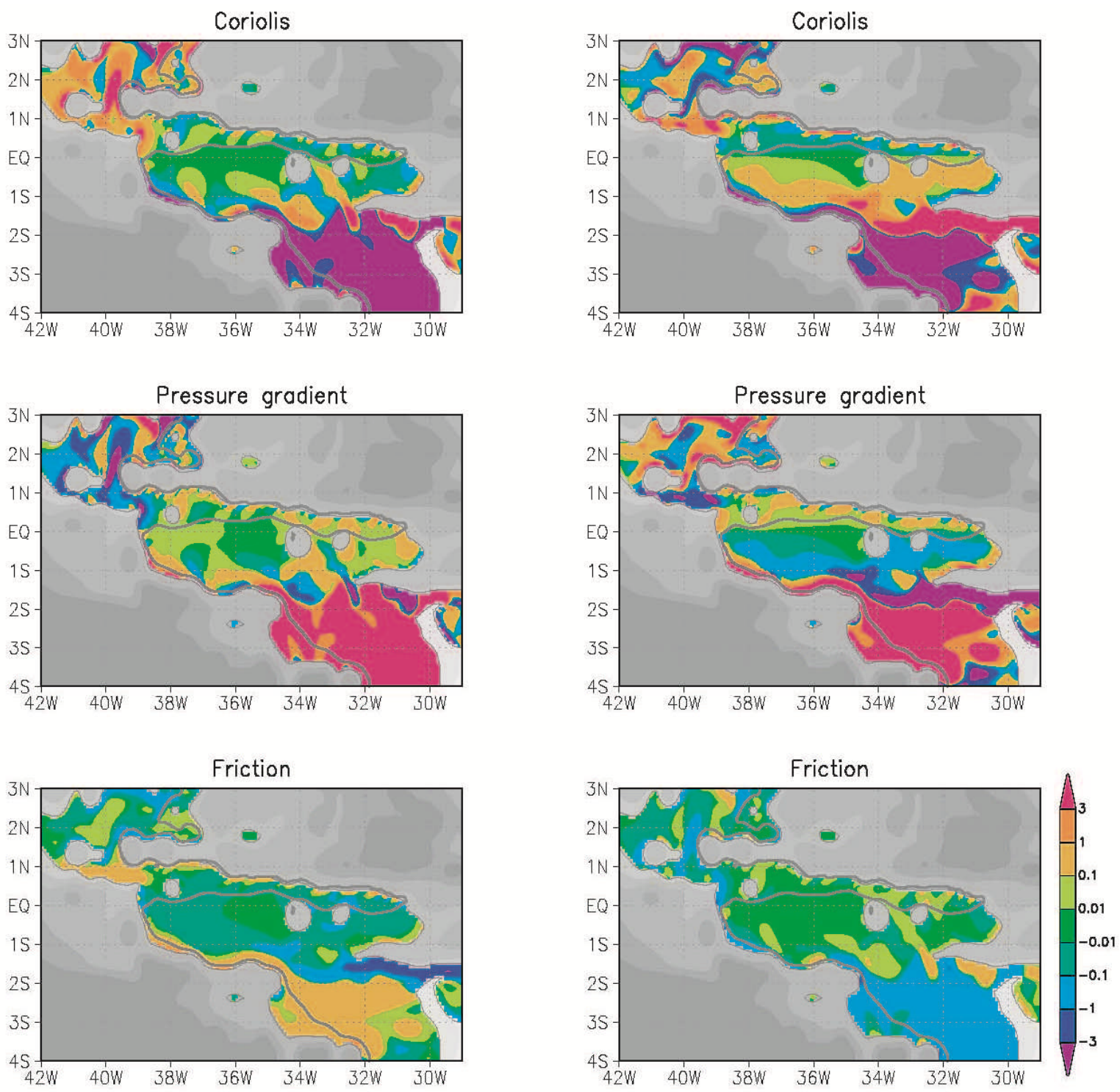

Figure 7. Momentum balances for frictional geostrophic simulation. (left) Zonal component. (right) Meridional component. Positive values represent an accelerating force in the eastward or northward direction. Nonuniform contour spacing is used to reveal features on different scales. Contours are the same for all plots, in units of $10^{-7} \mathrm{~m} \mathrm{~s}^{-2}$. A transport stream function contour line has been added to all plots for cross reference. 

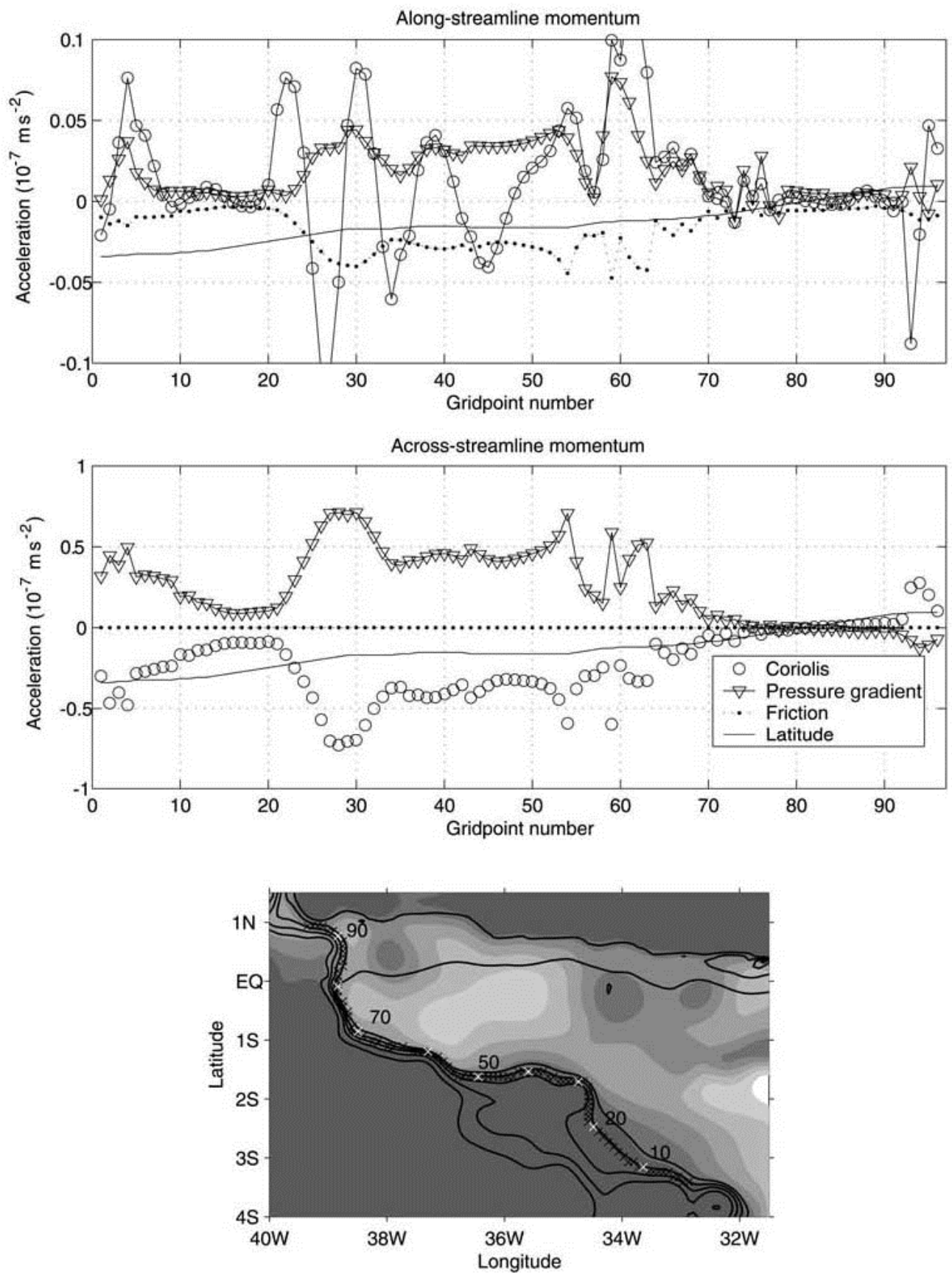

Figure 8. Momentum terms along a streamline, frictional geostrophic model. At each point the momentum terms are projected onto the along- and across-streamline directions. A positive quantity in the along-stream direction represents a driving force. A positive quantity in the across-stream plot represents a force pushing to the right. Along-stream Coriolis is not zero because of the way the staggered Arakawa $\mathrm{C}$ grid is used in the discretization of $\mathrm{FG}$. 


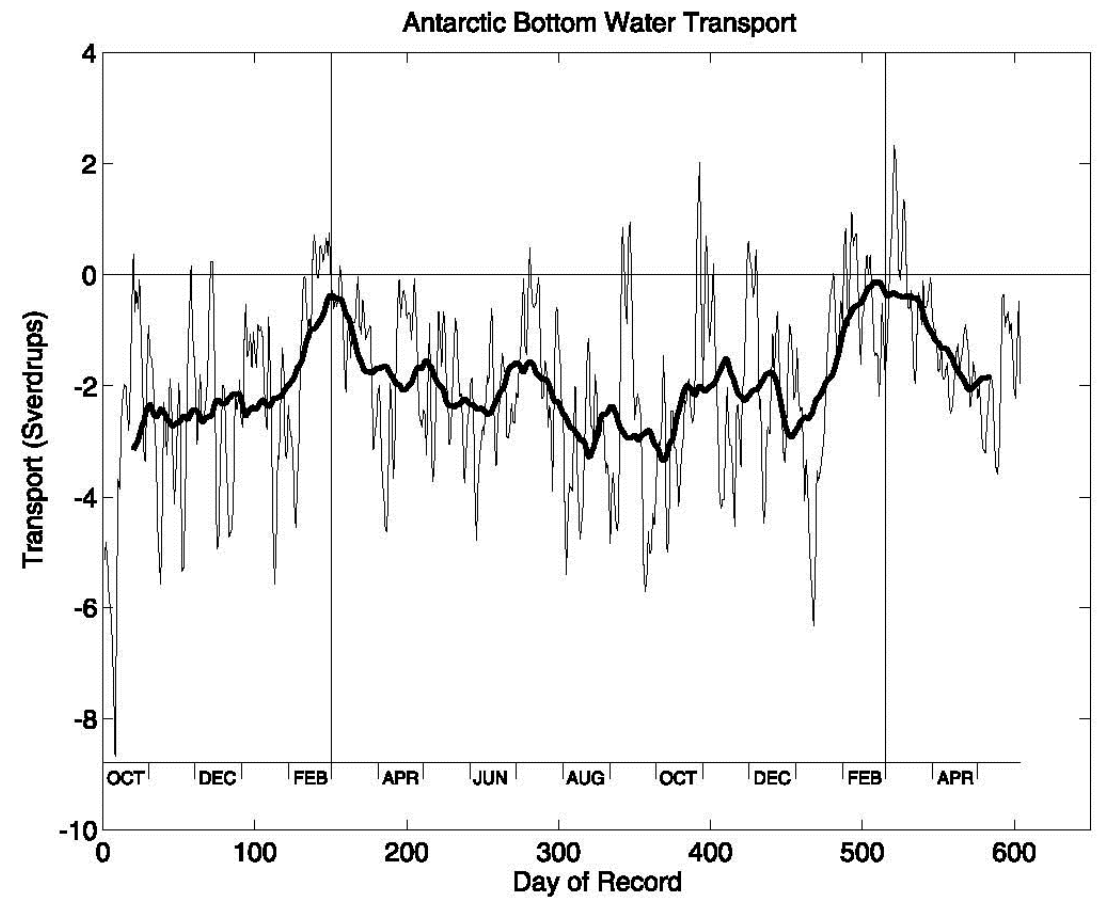

Figure 9. Time-dependent flux as measured by Hall et al. [1997]. The thin line is daily transport, and the thick line is the 40 day boxcar average. Negative values indicate westward flow. The line marking zero transport and the vertical lines marking the beginning of March each year have been added to emphasize the annual signal.

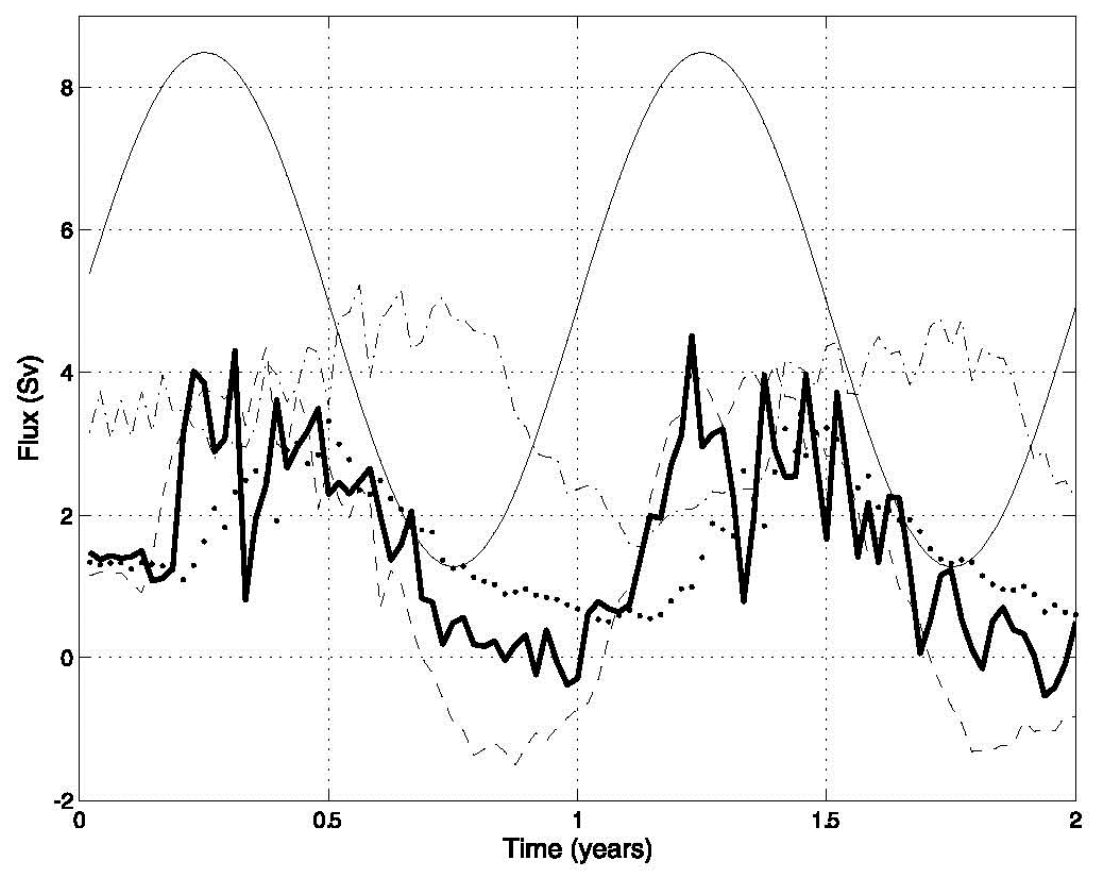

Figure 10. Time-dependent flux as predicted by shallow water theory. The thin solid line shows inflow conditions, the dash-dotted line shows outflow eastward, the dashed line shows flow into the equatorial basin, the dotted line shows flow out of the equatorial basin, and the thick solid line shows flow across Hall et al. [1997] mooring locations. 


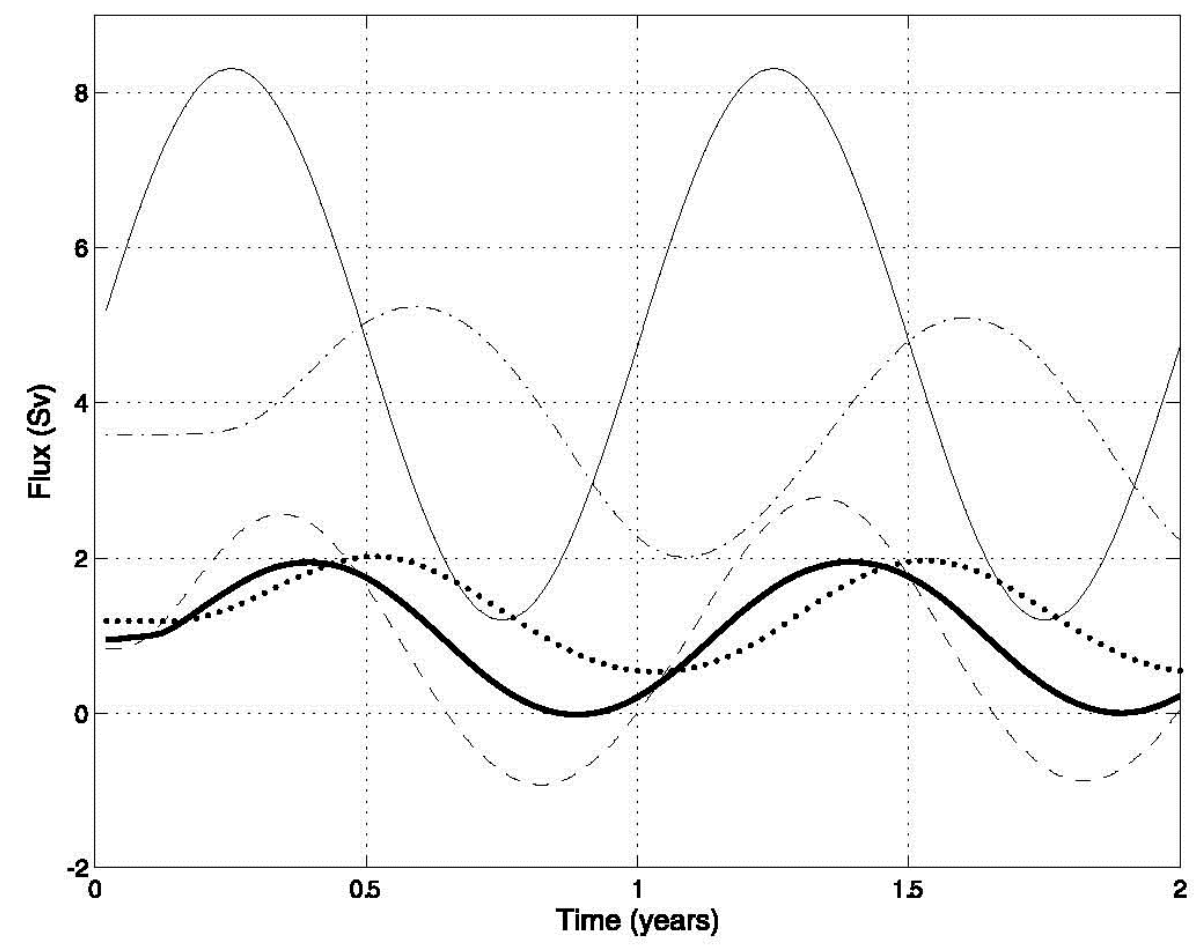

Figure 11. Time-dependent flux as predicted by the FG model. The thin solid line shows inflow conditions, the dash-dotted line shows outflow eastward, the dashed line shows flow into the equatorial basin, the dotted line shows flow out of the equatorial basin, and the thick solid line shows flow across Hall et al. [1997] mooring locations.

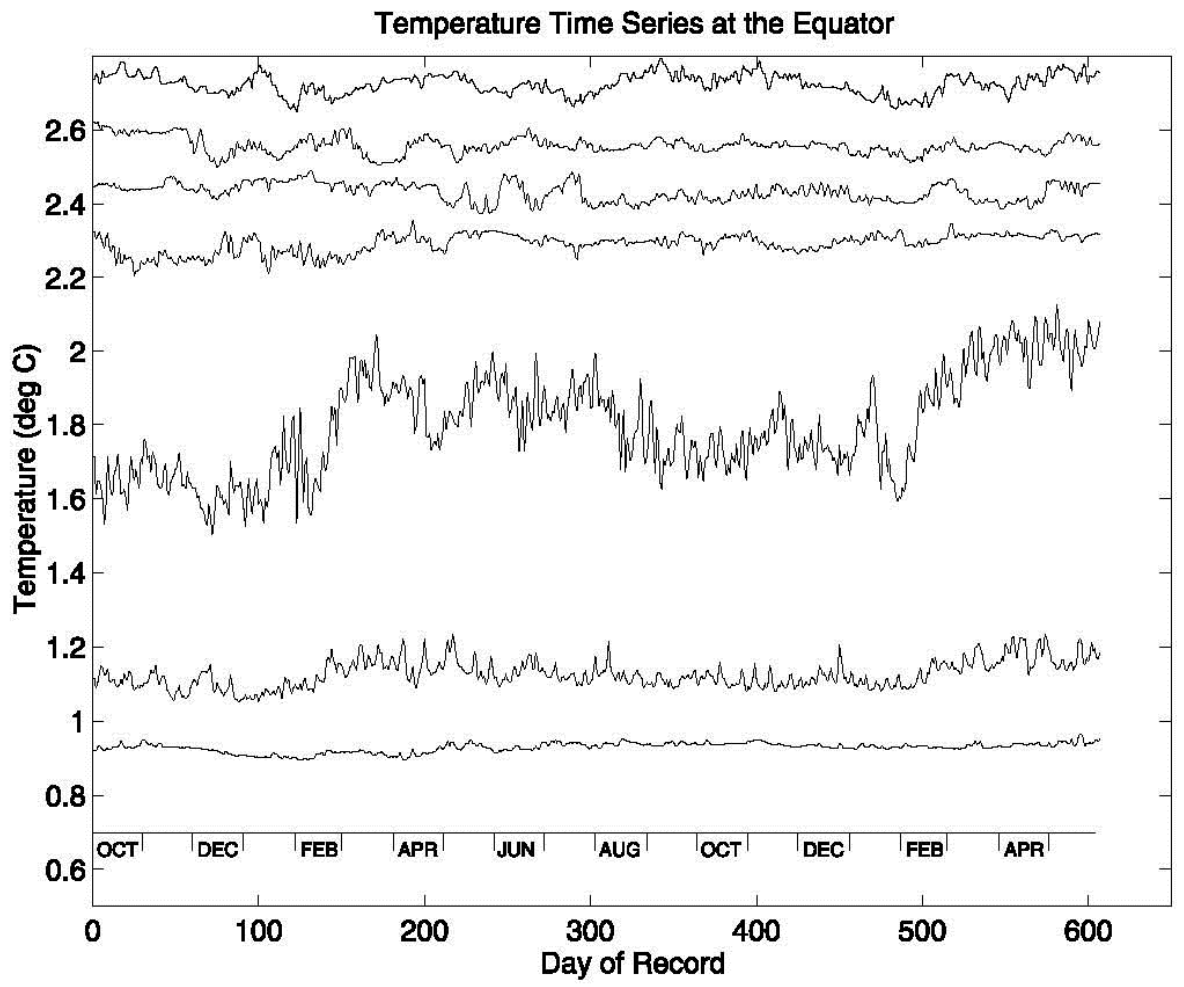

Figure 12. Time series of temperature on the equator at $36^{\circ} \mathrm{W}$ as measured by Hall et al. [1997]. Depths of measurements are (from warmest to coldest) 2993, 3293, 3593, 3892, 4093, 4292, and $4485 \mathrm{~m}$. 


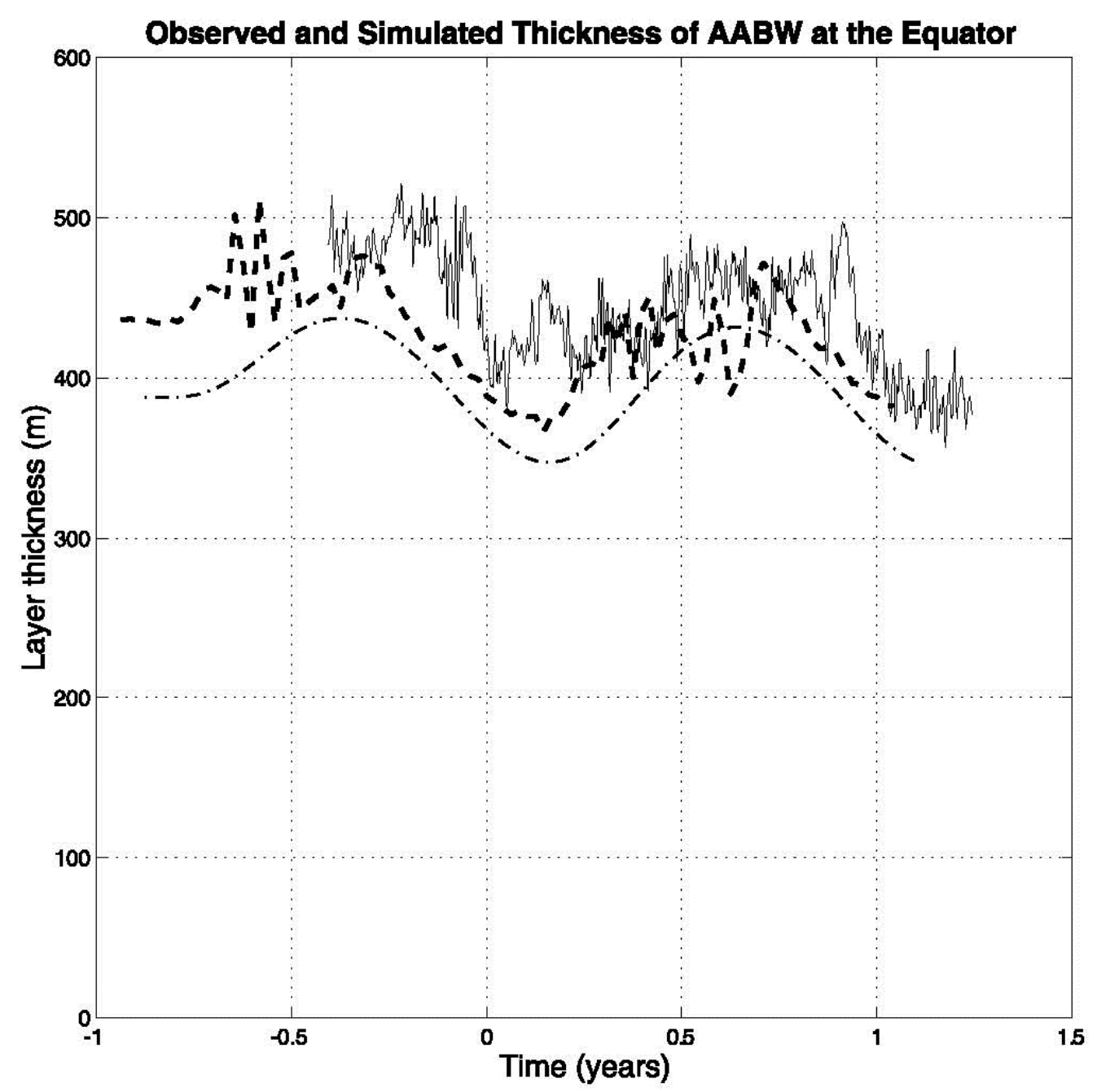

Figure 13. Depth of Antarctic Bottom Water (AABW) at $36^{\circ} \mathrm{W}$ on the equator. The thin solid line is calculated from observations. The thick dashed line shows the shallow water simulation. The thin dashdotted line shows the FG simulation. The depth of the $1.8^{\circ} \mathrm{C}$ isotherm is assumed to be the surface of $\mathrm{AABW}$ and is calculated from the temperature data of Hall et al. [1997]. Time $(T)=0$ coincides with the minimum transport annually: 1 March in observations, $T=0.96$ years in the shallow water simulation, and $T=0.90$ years in the FG simulation. The depth of the ocean floor at this location is $4536 \mathrm{~m}$. 